13

\title{
Аналитическая модель атомно-слоевого осаждения тонких пленок на стенки цилиндрических отверстий с высоким аспектным отношением
}

\author{
(C) А.В. Фадеев, К.В. Руденко \\ Физико-технологический институт РАН, \\ 117218 Москва, Россия \\ e-mail: AlexVFadeev@gmail.com
}

(Поступило в Редакцию 28 декабря 2017г.)

Предложена теоретическая модель, предсказывающая профиль толщины пленки, выращиваемой на стенках высокоаспектного цилиндрического отверстия методом атомно-слоевого осаждения. Модель дает возможность рассчитать критическое время подачи прекурсора, необходимое для конформного покрытия стенок отверстия. Получена аналитическая формула, позволяющая оценить минимальное время подачи прекурсора в зависимости от параметров технологического процесса.

DOI: $10.21883 /$ JTF.2018.08.46319.2625

\section{Введение}

В настоящее время активно развивается технология $3 D$-интеграции чипов интегральных схем различного назначения. Объединяющим элементом является пластина с цилиндрическими отверстиями, которые заполняются металлическими вставками. Аспектное отношение (отношение глубины к ширине) этих отверстий находится в диапазоне 5-20. Технология заполнения предполагает формирование проводящего лайнера тонкой пленки, конформно нанесенной на стенку отверстия. Аналогичные технологии используются при создании проводящих вставок в отверстиях межслойного диэлектрика систем многоуровневой металлизации ультрабольших интегральных схем (УБИС), где конформная тонкая пленка на стенке отверстия нужна как барьер диффузии меди.

Весьма впечатляющие результаты были достигнуты [1] на структурах интегральных конденсаторов большой емкости на основе пористого кремния, при заполнении высокоаспектных пор последовательно слоями диэлектрика и металла. В таких порах аспектное отношение может достигать значений 100-1000. Таким образом, конформное нанесение пленок на стенки отверстий с различным значением аспектных отношений является актуальной задачей.

Одной из наиболее перспективных технологий конформного нанесения тонких пленок на $3 D$-структуры является атомно-слоевое осаждение (ALD) [2]. Этот метод позволяет выращивать конформные пленки с жестким контролем толщины, который обеспечивается самонасыщением протекания поверхностных реакций на каждом ALD-цикле. Реакции, формирующие мономолекулярную пленку заданного состава с адсорбированным на поверхности первым прекурсором, прекращаются после того, как молекулы второго прекурсора (активные радикалы плазмы, молекулы воды) прореагируют со всеми молекулами адсорбата. Повторение ALD-циклов определяет общую толщину выращенной пленки.

На 3D-структурах с высоким аспектным отношением процессом, лимитирующим рост тонких пленок методом $\mathrm{ALD}$, как правило, является транспорт первого прекурсора в высокоаспектное отверстие. Поэтому исследование зависимости времени подачи металлорганических и неорганических прекурсоров, достаточного для образования конформной пленки, от величины аспектного отношения $3 D$-структур и коэффициента прилипания молекул прекурсора является предметом теоретических исследований ряда авторов [2-9]. Большинство работ в этой области представляет собой численное моделирование методом Монте-Карло (см., например, [4-6]). Из теоретических работ наиболее известной является работа [3], в основе модели которой используется формула для стационарного течения кнудсеновского газа по трубе, соединяющей две полости, а также равный единице коэффициент прилипания частиц прекурсора. В [3] не принимался во внимание поток частиц, отраженных от дна отверстия. В работах [7-9] предлагается кинетическая модель, согласно которой реагирующее вещество поступает в каналы наноструктуры по диффузионному закону и необратимо взаимодействует с активными узлами на стенках $3 D$-структуры. Граничные условия, используемые в данной модели, недостаточно обоснованы. В отличие от [7-9] в предлагаемой нами модели потоки частиц прекурсора на стенки и дно тренча определяются согласно молекулярно-кинетической теории, а степень заполнения элемента поверхности со временем подачи прекурсора описывается системой кинетических уравнений.

Целью настоящей работы является создание аналитической модели транспорта прекурсоров в цилиндрические отверстия и теоретическое исследование степени покрытия стенок отверстия в зависимости от параметров ALD-процесса и аспектного отношения исследуемой структуры. 


\section{1. Система кинетических уравнений}

Для количественного сравнения полученных нами результатов с другими авторами [5] будем исследовать рост пленки $\mathrm{HfO}_{2}$ методом атомно-слоевого осаждения из тетраэтилметиламиногафния (ТЕМАН) и паров воды. Как было показано нами ранее [10], время ALD-цикла в этом случае лимитируется временем подачи первого прекурсора (ТЕМАН). Поэтому оптимальный подбор времени экспозиции ТЕМАН позволяет, с одной стороны, уменьшить полное время процесса нанесения пленки, а с другой - снизить расход дорогостоящего прекурсора.

Оценки, проведенные в работе [10], показали, что при характерных параметрах ALD-процессов, применяемых при создании интегральных схем, длина свободного пробега молекул прекурсора значительно превышает диаметр цилиндрического отверстия, поэтому можно пренебречь взаимодействием между частицами внутри отверстий и учитывать только их взаимодействие со стенками.

Для построения кинетической модели, описывающей зависимость степени покрытия стенок цилиндрического

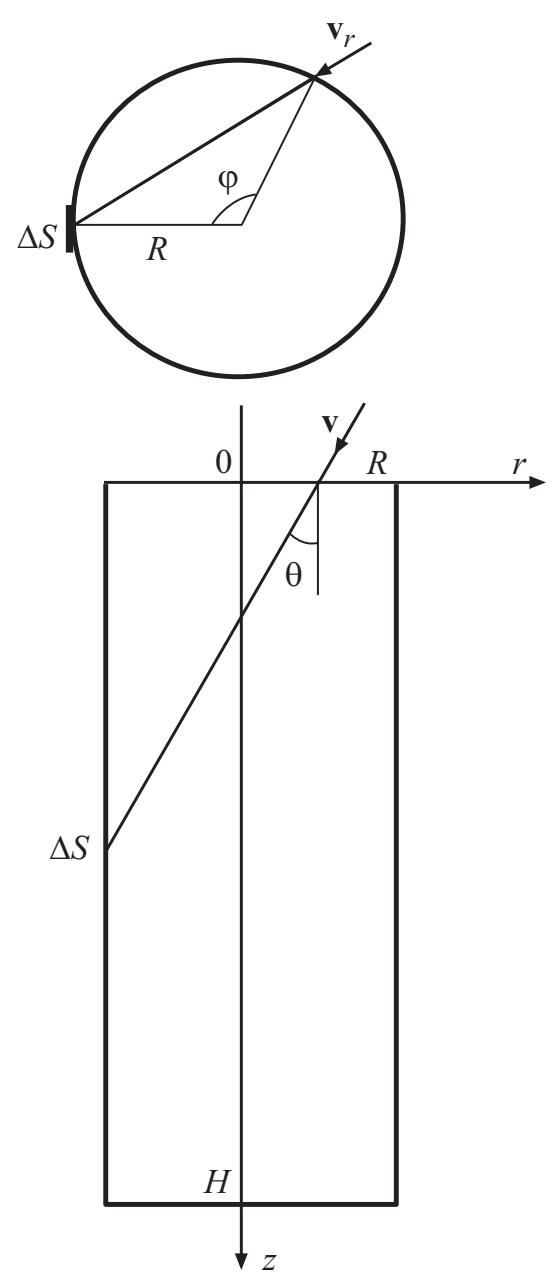

Рис. 1. Поток частиц из газовой фазы на элемент боковой стенки цилиндрического отверстия. отверстия от времени подачи прекурсора, вводятся следующие допущения:

- параметры газа, подаваемого в камеру реактора, а также размеры цилиндрического отверстия соответствуют молекулярному режиму течения газа внутри цилиндрического отверстия;

- концентрация молекул прекурсора в газовой фазе над плоской поверхностью постоянна, а их распределение по скоростям подчиняется распределению Максвелла;

- возможность адсорбции второго монослоя равна нулю; - при взаимодействии с поверхностью частица прекурсора может либо отразиться от нее по закону косинуса с вероятностью

$$
p=\Theta+(1-\Theta)(1-s)=1-s(1-\Theta),
$$

где $\Theta$ - степень покрытия стенок отверстия, а $s-$ коэффициент прилипания молекул прекурсора, либо прилипнуть с вероятностью $1-p$;

- отраженная частица приобретает температуру поверхности;

- пренебрегается возможностью поверхностной диффузии адсорбированных частиц.

С учетом сделанных предположений кинетическое уравнение, описывающее зависимость степени покрытия стенок отверстия от времени подачи прекурсора, имеет вид

$$
n_{0} \frac{d \Theta}{d t}=\left(J_{e}+J_{r}\right) s(1-\Theta),
$$

где $n_{0}-$ поверхностная концентрация прекурсора в адсорбированном монослое, а $J_{e}$ и $J_{r}$ - плотности потоков частиц на площадку $d S$ цилиндрического отверстия из газовой фазы и за счет отражений от других частей отверстия соответственно.

При расчетах использованы характерные для ТЕМАН параметры ALD-процесса [5]: температура подложки $T_{s}=270^{\circ} \mathrm{C}$ и давление $P=1$ mTorr.

\section{1. Плотность внешнего потока частиц на боковую поверхность цилиндрического отверстия $\left(J_{e s}\right)$}

Плотность потока частиц на элемент поверхности цилиндрического отверстия определяется как число ударов о поверхность площадью $\Delta S$ за время $\Delta t$ :

$$
d \hat{N}=d N_{v} \frac{v_{\perp} \Delta t \Delta S}{V}
$$

где $v_{\perp}-$ перпендикулярная площадке $\Delta S$ компонента скорости частиц, $d N_{v}$ - число частиц, имеющих скорость в диапазоне от $v$ до $v+d v$. Умножив и поделив (3) на полное число частиц $N$, получим выражение для плотности потока частиц на площадку $\Delta S$ за время $\Delta t$ :

$$
\begin{aligned}
d \hat{N} & =v_{\perp} \Delta S \Delta t \frac{N}{V} \frac{d N_{v}}{N}=\left[\frac{N}{V}=n, \frac{d N_{v}}{N}=f(v) d v\right] \\
& =v_{\perp} \Delta S \Delta \operatorname{tn} f(v) d v .
\end{aligned}
$$


Согласно рис. 1, о площадку $\Delta S$ на боковой стенке цилиндрического отверстия, находящуюся на расстоянии $z$ от поверхности, за время $\Delta t$ ударятся те частицы, направление скорости которых лежит в диапазоне углов $\theta \in\left(0 ; \theta_{\max }\right]$. Связь $\theta_{\max }$ и перпендикулярной компоненты скорости частиц с параметрами отверстия определяются соотношениями:

$$
\operatorname{tg}\left(\theta_{\max }\right)=\frac{2 R \sin (\varphi / 2)}{z}, \quad v_{\perp}=v \sin \theta \sin \frac{\varphi}{2} .
$$

Будем полагать максвелловское распределение частиц по скоростям, находящихся над плоской поверхностью. Плотность их потока на боковую стенку цилиндрического отверстия будет иметь вид

$$
\begin{aligned}
& J_{e s}(z)=\left[\frac{1}{2}\right] n_{g}\left(\frac{m}{2 \pi k T_{g}}\right)^{3 / 2} \\
& \quad \times \int_{0}^{\infty} v^{2} d v \int_{0}^{2 \pi} d \varphi \int_{0}^{\theta_{\max }} \exp \left(-\frac{m v^{2}}{2 k T_{g}}\right) v \sin \theta \sin \frac{\varphi}{2} \sin \theta d \theta \\
& =n_{g} \sqrt{\frac{k T_{g}}{2 \pi m}} \frac{z^{2}+2 R^{2}-z \sqrt{z^{2}+4 R^{2}}}{2 R \sqrt{z^{2}+4 R^{2}}} \\
& \approx|R \ll z| \approx n_{g} \sqrt{\frac{k T_{g}}{2 \pi m}}\left(\frac{R}{z}\right)^{3}=\left(\frac{R}{z}\right)^{3} J_{f},
\end{aligned}
$$

где

$$
J_{f}=n_{g} \sqrt{\frac{m}{2 \pi k T_{g}}} \int_{0}^{\infty} v_{z} \exp \left(-\frac{m v_{z}^{2}}{2 k T_{g}}\right) d v_{z}=n_{g} \sqrt{\frac{k T_{g}}{2 \pi m}}
$$

- плотность потока частиц на плоскую поверхность. Коэффициент, стоящий в квадратных скобках, учитывает, что частицы могут ударяться о площадку $\Delta S$ только с одной стороны.

\section{2. Плотность внешнего потока частиц на дно цилиндрического отверстия $\left(J_{e b}\right)$}

О площадку площадью $\Delta S$ на дне цилиндрического отверстия на расстоянии $r$ от его центра за время $\Delta t$ (рис. 2) ударятся те частицы, направление скорости которых лежит в диапазоне углов $\left(0 ; \theta_{\max }^{\prime}\right]$. Максимальный угол и перпендикулярная дну компонента скорости частиц в этом случае определяются выражениями

$$
\operatorname{tg}\left(\theta_{\max }^{\prime}\right)=\frac{\sqrt{R^{2}+r^{2}-2 R r \cos \varphi}}{H}, \quad v_{\perp}=v \cos \theta .
$$

В результате, согласно (4), плотность потока частиц газа, находящегося над плоской поверхностью, на дно

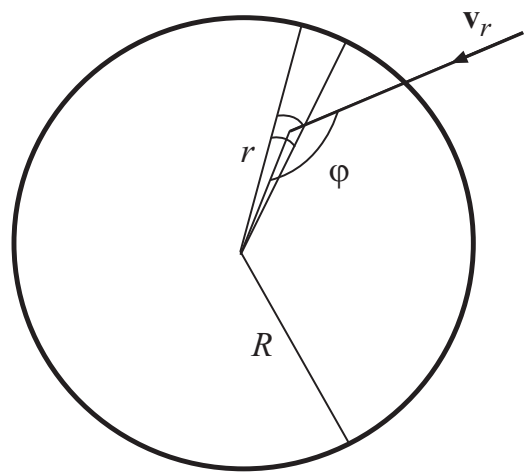

Рис. 2. Поток частиц из газовой фазы на элемент дна цилиндрического отверстия.

цилиндрического отверстия можно вычислить по формуле

$$
\begin{aligned}
J_{e b}(r)= & n_{g}\left(\frac{m}{2 \pi k T_{g}}\right)^{3 / 2} \int_{0}^{\infty} v^{2} d v \\
& \times \int_{0}^{2 \pi} d \varphi \int_{0}^{\theta_{\max }} \exp \left(-\frac{m v^{2}}{2 k T_{g}}\right) v \cos \theta \sin \theta d \theta \\
= & n_{g} \sqrt{\frac{k T_{g}}{2 \pi^{3} m}} \int_{0}^{2 \pi} \frac{\frac{1}{2}\left(R^{2}+r^{2}-2 R r \cos \varphi\right)}{H^{2}+R^{2}+r^{2}-2 R r \cos \varphi} d \varphi \\
\approx & \mid R \ll{ }^{2} \approx \\
= & \left(\frac{R^{2}+r^{2}}{H^{2}}\right) J_{f} .
\end{aligned}
$$

Сравнивая потоки частиц из газовой фазы, можно ожидать, что при больших аспектных отношениях дно цилиндрического отверстия заполниться гораздо быстрее, чем его боковая поверхность, и станет источником отраженных частиц даже при коэффициенте прилипания, равном единице.

\section{3. Плотность потока частиц на боковую стенку цилиндрического отверстия, индуцированная отражением от его дна $\left(J_{b s}\right)$}

Количество частиц, испускаемых площадкой $\Delta S$ в телесный угол $d \Omega$ под углом $\theta$ к нормали в единицу времени, определяется через поток частиц $\Phi$ выражением

$$
J=\frac{d \Phi}{d \Omega \Delta S_{\perp}}=\frac{d \Phi}{d \Omega \Delta S \cos \theta} \Rightarrow d \Phi=J d \Omega \Delta S \cos \theta .
$$

Для ламбертовского источника $J=$ const. Будем предполагать, что частицы при отражении от поверхности 


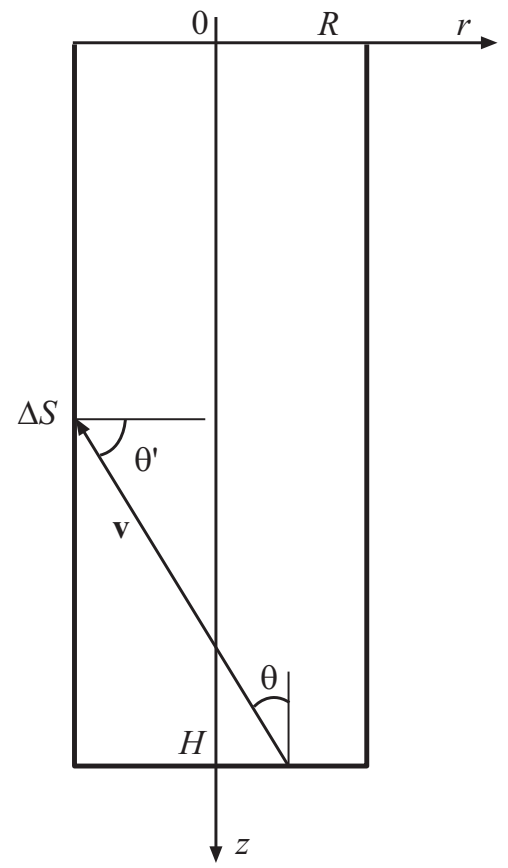

Рис. 3. Поток частиц на элемент боковой стенки цилиндрического отверстия за счет частиц, отраженных от его дна.

подчиняются закону Ламберта (закон косинуса). Также будем предполагать, что распределение отраженных частиц по скоростям, будучи дополненным таким же числом частиц, летящих в сторону стенки, подчиняется закону Максвелла с температурой, равной температуре стенок цилиндрического отверстия $\left(T_{s}\right)$, и вероятность осаждения второго монослоя равна нулю.

Число молекул, ударившихся в единицу времени об элемент поверхности $r d r d \varphi$ на дне цилиндрического отверстия, равно $J_{b}(r) r d r d \varphi$, где $J_{b}(r)$ - плотность потока частиц на дно цилиндрического отверстия. Из них внутри телесного угла $\Delta \Omega$ по направлению к площадке $\Delta S$ на боковой стенке цилиндрического отверстия, согласно закону косинуса, отразится

$$
p(r, H) J_{b}(r) r d r d \varphi \frac{\Delta \Omega}{\pi} \cos \theta
$$

частиц (рис. 3), где $p(r, H)-$ вероятность отражения частиц от элемента дна цилиндрического отверстия (1). Учитывая, что

$$
\begin{aligned}
\Delta \Omega & =\frac{\Delta S \cos \theta^{\prime}}{(H-z)^{2}+\left(R^{2}+r^{2}-2 R r \cos \varphi\right)}, \\
\cos \theta & =\frac{H-z}{\sqrt{(H-z)^{2}+\left(R^{2}+r^{2}-2 R r \cos \varphi\right)}}, \\
\cos \theta^{\prime} & =\frac{R-r \cos \varphi}{\sqrt{(H-z)^{2}+\left(R^{2}+r^{2}-2 R r \cos \varphi\right)}},
\end{aligned}
$$

после интегрирования по площади дна получим выражение для плотности потока частиц на площадку
$\Delta S$ боковой стенки цилиндрического отверстия за счет отраженных от дна частиц:

$$
\begin{aligned}
J_{b s}(z)= & \frac{H-z}{\pi} \int_{0}^{R}(1-s(1-\Theta(r, H))) J_{b}(r) r d r \\
& \times \int_{0}^{2 \pi} \frac{R-r \cos \varphi}{\left((H-z)^{2}+\left(R^{2}+r^{2}-2 R r \cos \varphi\right)\right)^{2}} d \varphi .
\end{aligned}
$$

Поток частиц на дно цилиндрического отверстия $\left(J_{b}\right)$ определяется суммой внешнего потока $\left(J_{e b}\right)$ и потока, отраженного от боковых стенок цилиндрического отверстия $\left(J_{s b}\right)$. После интегрирования по полярному углу $\varphi$ окончательно получим выражение для потока $J_{b s}(z)$ :

$$
\begin{aligned}
& J_{b s}(z)=2 R(H-z) \int_{0}^{R}(1-s(1-\Theta(r, H))) \\
& \times\left(J_{e b}(r) \sqrt{\frac{T_{s}}{T_{g}}}+J_{s b}(r)\right) \\
& \times \frac{(H-z)^{2}+R^{2}-r^{2}}{\left((H-z)^{4}+2(H-z)^{2}\left(R^{2}+r^{2}\right)+\left(R^{2}-r^{2}\right)^{2}\right)^{3 / 2}} r d r .
\end{aligned}
$$

Корень $\sqrt{\frac{T_{s}}{T_{g}}}$ в скобках учитывает, что молекулы после удара о поверхность приобретают температуру подложки.

\section{4. Плотность потока частиц на дно цилиндрического отверстия, индуцированная отражением от его стенки $\left(J_{s b}\right)$}

Пусть $J_{s}(z)-$ плотность потока частиц на элемент боковой стенки цилиндрического отверстия, расположенный на расстоянии $z$ от поверхности. Поток $J_{s}(z)$ определяется суммой внешнего потока $\left(J_{e s}\right)$, потока, отраженного от дна цилиндрического отверстия $\left(J_{b s}\right)$, и потока, обусловленного другими частями боковой стенки цилиндрического отверстия $\left(J_{s s}\right)$. Число молекул, ударившихся в единицу времени об элемент поверхности $R d \varphi d z$ боковой стенки, равно $J_{s}(z) R d \varphi d z$. Из них внутри телесного угла $\Delta \Omega$ по направлению к площадке $\Delta S$, согласно закону косинуса, отразится

$$
p(R, z) J_{s}(z) R d \varphi d z \frac{\Delta \Omega}{\pi} \cos \theta^{\prime}
$$

частиц (рис. 4).

В силу симметрии задачи на любой участок кольца $r d r d \varphi$, расположенного на дне, попадает одинаковое число частиц, отраженных боковой стенкой цилиндрического отверстия. Поэтому будем считать, что указанный 


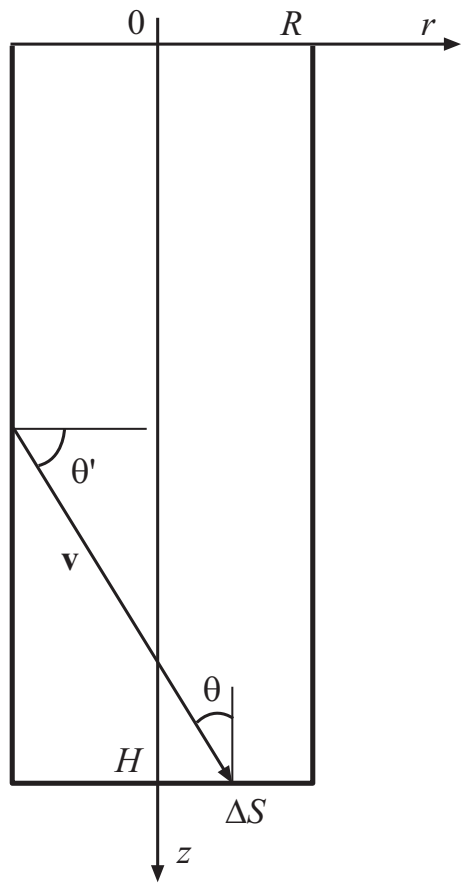

Рис. 4. Поток частиц на элемент дна цилиндрического отверстия за счет частиц, отраженных от его боковой стенки.

элемент имеет угловую координату $\varphi=0$. Принимая во внимание выражения для телесного угла:

$$
\Delta \Omega=\frac{\Delta S \cos \theta}{(H-z)^{2}+\left(R^{2}+r^{2}-2 R r \cos \varphi\right)},
$$

а также косинусов соответствующих углов (12), после интегрирования по поверхности стенок цилиндрического отверстия получим плотность потока частиц, отраженных от боковой стенки, на дно цилиндрического отверстия:

$$
\begin{aligned}
& J_{s b}(r)=\int_{0}^{H}(1-s(1-\Theta(R, z))) J_{s}(z) R d z \\
& \times \int_{0}^{2 \pi} d \varphi \frac{\cos \theta}{(H-z)^{2}+\left(R^{2}+r^{2}-2 R r \cos \varphi\right)} \frac{1}{\pi} \cos \theta^{\prime} \\
& =2 R^{2} \int_{0}^{H}(1-s(1-\Theta(R, z))) \\
& \times\left(J_{e s}(z) \sqrt{\frac{T_{s}}{T_{g}}}+J_{b s}(z)+J_{s s}(z)\right) \\
& \times \frac{\left((H-z)^{2}+R^{2}-r^{2}\right)(H-z)}{\left((H-z)^{4}+2(H-z)^{2}\left(R^{2}+r^{2}\right)+\left(R^{2}-r^{2}\right)^{2}\right)^{3 / 2}} d z .
\end{aligned}
$$

\section{5. Плотность потока частиц на элемент боковой стенки цилиндрического отверстия, индуцированная отражением от других частей стенки $\left(J_{s s}\right)$}

Число частиц, ударившихся в единицу времени об элемент поверхности боковой стенки с координатой $z_{1}$, равно $J_{s}\left(z_{1}\right) R d \varphi d z_{1}$, где $J_{s}=J_{e s}+J_{b s}+J_{s s}$. Из них внутри телесного угла $\Delta \Omega$ по направлению к площадке $\Delta S$, находящейся на боковой стенке с координатой $z$, отразится

$$
p\left(R, z_{1}\right) J_{s}\left(z_{1}\right) R d \varphi d z_{1} \frac{\Delta \Omega}{\pi} \cos \theta_{1}
$$

частиц. Аналогично предыдущему разделу будем полагать, что элемент поверхности боковой стенки с координатой $z$ имеет угловую координату $\varphi=0$. Принимая во внимание выражения для элемента телесного угла и косинусов соответствующих углов (рис. 5):

$$
\begin{aligned}
& \Delta \Omega=\frac{\Delta S \cos \theta}{\left(z-z_{1}\right)^{2}+(2 R \sin (\varphi / 2))^{2}}, \\
& \cos \theta_{1}=\cos \theta=\frac{2 R \sin ^{2}(\varphi / 2)}{\sqrt{\left(z-z_{1}\right)^{2}+4 R^{2} \sin ^{2}(\varphi / 2)}},
\end{aligned}
$$

после интегрирования по $z_{1}$ получим выражение для плотности потока частиц, отраженных от боковой стенки

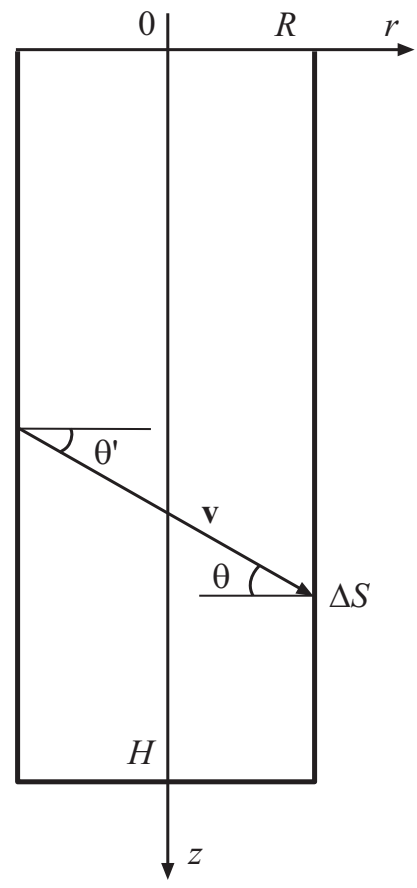

Рис. 5. Поток частиц на элемент поверхности боковой стенки цилиндрического отверстия за счет переотражений от стенки. 
и попавших на нее на расстоянии $z$ от поверхности:

$$
\begin{aligned}
J_{s s}(z)= & \int_{0}^{H}\left(1-s\left(1-\Theta\left(R, z_{1}\right)\right)\right) J_{s}\left(z_{1}\right) R d z_{1} \\
& \times \int_{0}^{2 \pi} d \varphi \frac{\cos \theta}{\left(z-z_{1}\right)^{2}+(2 R \sin (\varphi / 2))^{2}} \frac{1}{\pi} \cos \theta_{1} \\
= & \frac{1}{2 R} \int_{0}^{H}\left(1-s\left(1-\Theta\left(R, z_{1}\right)\right)\right) \\
& \times\left(J_{e s}\left(z_{1}\right) \sqrt{\frac{T_{s}}{T_{g}}}+J_{b s}\left(z_{1}\right)+J_{s s}\left(z_{1}\right)\right) \\
& \times\left(1-\frac{\left|z-z_{1}\right|\left(\left(z-z_{1}\right)^{2}+6 R^{2}\right)}{\left(\left(z-z_{1}\right)^{2}+4 R^{2}\right)^{3 / 2}}\right) d z_{1} .
\end{aligned}
$$

\section{2. Численное решение системы кинетических уравнений}

Расчеты, проведенные на основании выражений (6), (9), (14), (17), (20) для потоков, показывают, что наименьший результирующий поток прекурсора приходится на боковую стенку цилиндрического отверстия вблизи ее дна. Поэтому для определения минимального времени подачи прекурсора (критическое время), необходимого для конформного покрытия стенок цилиндрического отверстия, следует требовать, чтобы вблизи основания стенки цилиндрического отверстия степень покрытия превышала наперед заданную величину $(\eta)$.

Система интегродифференциальных уравнений

$$
\left\{\begin{array}{l}
n_{0} \frac{d \Theta(R, z)}{d t}=\left(J_{e s}(z)+J_{s s}(z)+J_{b s}(z)\right) s(1-\Theta(R, z)), \\
n_{0} \frac{d \Theta(r, H)}{d t}=\left(J_{e b}(r)+J_{s b}(r)\right) s(1-\Theta(r, H)),
\end{array}\right.
$$

описывающих степень заполнения поверхности цилиндрического отверстия, решалась численно. Для этого временной интервал разбивался на элементы $\Delta t$. На первом временном интервале в системе (21) отраженные потоки полагались равными нулю. Также в приближении нулевого покрытия определялись выражения для начальных отраженных потоков:

$$
\begin{aligned}
& J_{b s}(z)=2 R(H-z) \sqrt{\frac{T_{s}}{T_{g}}} \int_{0}^{R}(1-s) J_{e b}(r) \\
& \times \frac{(H-z)^{2}+R^{2}-r^{2}}{\left((H-z)^{4}+2(H-z)^{2}\left(R^{2}+r^{2}\right)+\left(R^{2}-r^{2}\right)^{2}\right)^{3 / 2}} r d r,
\end{aligned}
$$

$$
\begin{aligned}
& J_{s b}(r)=4 \pi R^{2} \sqrt{\frac{T_{s}}{T_{g}}} \int_{0}^{H}(1-s) J_{e s}(z) \\
& \times \frac{\left((H-z)^{2}+R^{2}-r^{2}\right)(H-z)}{\left((H-z)^{4}+2(H-z)^{2}\left(R^{2}+r^{2}\right)+\left(R^{2}-r^{2}\right)^{2}\right)^{3 / 2}} d z, \\
& J_{s s}(z)=\frac{\pi}{R} \sqrt{\frac{T_{s}}{T_{g}}} \int_{0}^{H}(1-s) J_{e s}\left(z_{1}\right) \\
& \times\left(1-\frac{\left|z-z_{1}\right|\left(\left(z-z_{1}\right)^{2}+6 R^{2}\right)}{\left(\left(z-z_{1}\right)^{2}+4 R^{2}\right)^{3 / 2}}\right) d z_{1} . \quad(22)
\end{aligned}
$$

На следующем временном интервале решение системы уравнений (21) осуществлялось при значении потоков и степени заполнения, вычисленной на предыдущем временном интервале. Далее процесс повторяется до тех пор, пока $\Theta_{\min }<\eta$, или до исчерпания заданного времени подачи прекурсора. Для количественного сравнения с результатами [5] степень конформности растущей пленки выбиралась равной $0.99(\eta=0.99)$.

На рис. 6 представлены кривые зависимости критического времени подачи прекурсора ТЕМАН от аспектного отношения цилиндрического отверстия в двойном логарифмическом масштабе аналогично работе [6]. Критическое время на рис. 6 нормировано на время покрытия плоской поверхности для коэффициента прилипания $s=1$. Эти кривые качественно согласуются с кривыми, полученными в работе [6] для тренчей (канавка прямоугольной формы). Для количественного сравнения результатов на рисунке символами также приведены значения, взятые из работы [5], полученные методом Монте-Карло. В [5] приведены нормированные величины на значение критического времени, соответствующего

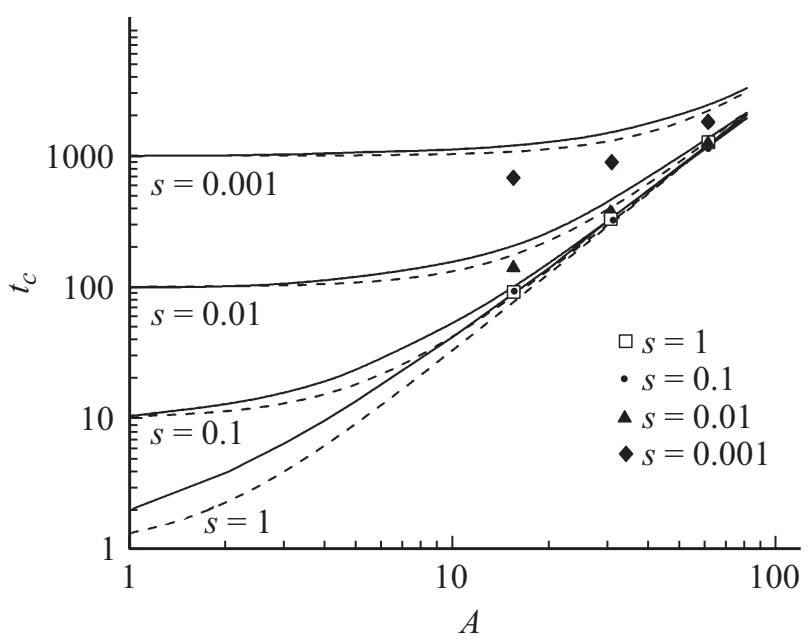

Рис. 6. Расчетные (сплошные кривые) и аналитические (штриховые кривые) зависимости критического времени покрытия стенок цилиндрического отверстия от аспектного отношения при различных значениях коэффициента прилипания. Значения, отмеченные точками, взяты из работы [5]. 
аспектному отношению $A=15$ и коэффициенту прилипания $s=1$. Для сравнения, критические времена из работы [5] были перенормированы на величину, необходимую для совпадения результатов при $A=15$ и $s=1$.

При больших значениях коэффициента прилипания точки, полученные в расчетах Монте-Карло [5], хорошо ложатся на численно проинтегрированные кривые (сплошные линии). С уменьшением коэффициента прилипания наблюдается некоторое систематическое завышение результатов расчетов, полученных в настоящей работе. Это может быть объяснено несколькими факторами. Уменьшение шага сетки и временного интервала при решении системы (21) снижает относительную погрешность результатов вычислений, завышающих результат, но сильно увеличивает время расчетов. При этом абсолютная расчетная ошибка растет с понижением коэффициента прилипания. Кроме того, некоторое расхождение результатов при малых $s$ может быть вызвано неуказанной точностью расчетов в работе [5]. Из общих соображений следует, что с уменьшением коэффициента прилипания время расчета в модели МонтеКарло значительно растет, а, следовательно, при его уменьшении будет расти ошибка вычислений.

На расчетных кривых (рис. 6) наблюдаются два асимптотических режима. В случае, когда $1 / s \gg A^{2}$ (мелкие отверстия, небольшие аспектные отношения), критическое время подачи прекурсора фактически не зависит от аспектного отношения. В обратном случае, когда $1 / s \ll A^{2}$ (глубокие отверстия), критическое время примерно пропорционально квадрату аспектного отношения и не зависит от коэффициента прилипания.

\section{3. Аналитическая формула для определения критического времени покрытия стенок цилиндрического отверстия}

Определение критического времени подачи прекурсора на основе системы (21) достаточно трудоемко и требует больших затрат расчетного времени. Поэтому получим аналитическую зависимость на основе исследования предельных случаев мелких и глубоких отверстий, иллюстрированных на рис. 6. Когда коэффициент прилипания отличен от единицы, частица в среднем испытает $N_{s}=1 / s$ столкновений, прежде чем прилипнет к стенке отверстия. Обозначим за $H_{s}$ среднюю глубину, на которую может продвинуться влетевшая в отверстие частица прежде, чем адсорбируется на стенке. Если $H_{s} \gg H$ (мелкие отверстия), то критическое время должно в основном определяться коэффициентом прилипания $s$ и не должно зависеть от аспектного отношения. В обратном случае $H_{s} \ll H$ (глубокое отверстие) должно наблюдаться постепенное продвижение монослоя растущей пленки в глубь отверстия и в основном определяться величиной аспектного отношения $A$.
Оценим глубину $H_{s}$. Плотность вероятности отражения частицы под углом $\theta$ в интервал $[0 ; \pi / 2]$ для случая рассеяния по закону косинуса определяется формулой

$$
\rho(\theta)=\cos \theta .
$$

Расстояние $\Delta z$, проходимое частицей между двумя последовательными столкновениями вдоль отверстия при заданном полярном угле, может быть получено из (19)

$$
\Delta z=\frac{2 R \sin ^{2}(\varphi / 2) \sqrt{\sin ^{2}(\varphi / 2)-\cos ^{2} \theta}}{\cos \theta},
$$

где значения угла $\theta$ ограничены положительностью подкоренного выражения. Тогда среднее значение $h$, проходимое частицей в глубь отверстия между двумя последовательными столкновениями со стенками, будет иметь вид

$$
h=\left\langle\int_{0}^{\pi / 2} \Delta z \rho(\theta) d \theta\right\rangle \approx 0.7 R .
$$

В (25) треугольные скобки означают усреднение по полярному углу $\varphi$.

C учетом (25) приближенное выражение для $H_{s}$ примет вид

$$
H_{s}^{2}=\left\langle\sum_{i=1}^{N_{s}} \sum_{j=1}^{N_{s}} h_{i} h_{j}\right\rangle \approx 0.5 R^{2} N_{s} .
$$

Результат, полученный по (26), оценочный, он не учитывает рост числа отражений по мере заполнения поверхности, а также уменьшение числа частиц за счет вылета из отверстия. В результате условие мелкого отверстия может быть записано

$$
H_{s} \sim \frac{R}{\sqrt{s}} \gg H \Rightarrow \frac{1}{s} \gg A^{2} .
$$

Полученный результат согласуется с выводами, сделанными из анализа расчетных кривых рис. 6. Положим, что критическое время покрытия стенок может быть записано в виде линейной комбинации двух предельных случаев

$$
t_{c}=\frac{k_{1}}{s}+k_{2} A^{2}
$$

Множитель $k_{1}$ может быть получен из условия, что для мелких отверстий $\left(1 / s \gg A^{2}\right)$ критическое время должно стремиться ко времени покрытия плоской поверхности до заданной степени покрытия растущей пленки $\eta$ :

$$
t_{c} \underset{A \rightarrow 0}{\longrightarrow} t_{f}=\frac{n_{0}}{s J_{f}} \ln \left(\frac{1}{1-\eta}\right) .
$$

В результате выражение для критического времени подачи прекурсора примет вид

$$
t_{c}=\frac{n_{0}}{s J_{f}} \ln \left(\frac{1}{1-\eta}\right)+k_{2} A^{2} .
$$


Для глубоких отверстий $\left(1 / s \ll A^{2}\right)$ будет происходить постепенное продвижение профиля в глубь отверстия, так как по мере заполнения поверхности его стенок будет расти среднее количество отражений частиц от стенок, а следовательно, и среднеквадратичное расстояние, проходимое ими в глубь отверстия.

Проводимость канала (поток газа, отнесенный к перепаду давлений) определяется обратной суммой проводимостей его частей [11]

$$
\frac{1}{c}=\frac{1}{c_{0}}+\frac{1}{c_{0}} \int_{0}^{z} \frac{3 d z}{8 R}=\frac{1}{c_{0}}+\frac{3 z}{8 R c_{0}},
$$

где $c_{0}$ - проводимость отверстия. В результате проводимость канала глубиной $z$ будет иметь вид

$$
c=\frac{c_{0}}{K(z)}, \quad K(z)=1+\frac{3 z}{8 R}
$$

Согласно формуле (32), поток газа будет уменьшаться по мере удаления от входного отверстия по закону

$$
\Phi(z)=\frac{J_{f} \pi R^{2}}{K(z)} .
$$

Учитывая, что число частиц, необходимое для монослойного покрытия элемента поверхности, равно $d N=n_{0} d S$, получим критическое время для монослойного покрытия боковых стенок отверстия

$$
d t=\frac{d N}{\Phi}=\frac{n_{0} d S}{\Phi}=\frac{K(z) n_{0} 2 \pi R d z}{J_{f} \pi R^{2}}
$$

После интегрирования по глубине отверстия получим выражение для времени заполнения стенок канала в случае $A \gg 1$ :

$$
t=\frac{2 n_{0}}{J_{f} R}\left(H+\frac{3 H^{2}}{16 R}\right) \approx \frac{3 n_{0}}{2 J_{f}} A^{2} .
$$

Объединяя формулы для мелкого и глубокого отверстий, окончательно получим аналитическое выражение для критического времени заполнения стенок цилиндрического отверстия

$$
t_{c}(A, s)=\frac{n_{0}}{J_{f}}\left(\frac{1}{s} \ln \left(\frac{1}{1-\eta}\right)+\frac{3}{2} A^{2}\right) .
$$

Данная формула с точностью до нормировочных множителей совпадает с выражением, полученным в работе [10] из анализа системы кинетических уравнений (21) для ALD-роста пленок в тренчах. Следует также заметить, что формула (36) аналогична аналитической зависимости, полученной в работах $[7,8]$ из решения диффузионной задачи, что также говорит в пользу ее справедливости. Формула (36) позволяет, не прибегая к длительным расчетам, оценить критическое время подачи прекурсора. Как можно видеть из рис. 6,

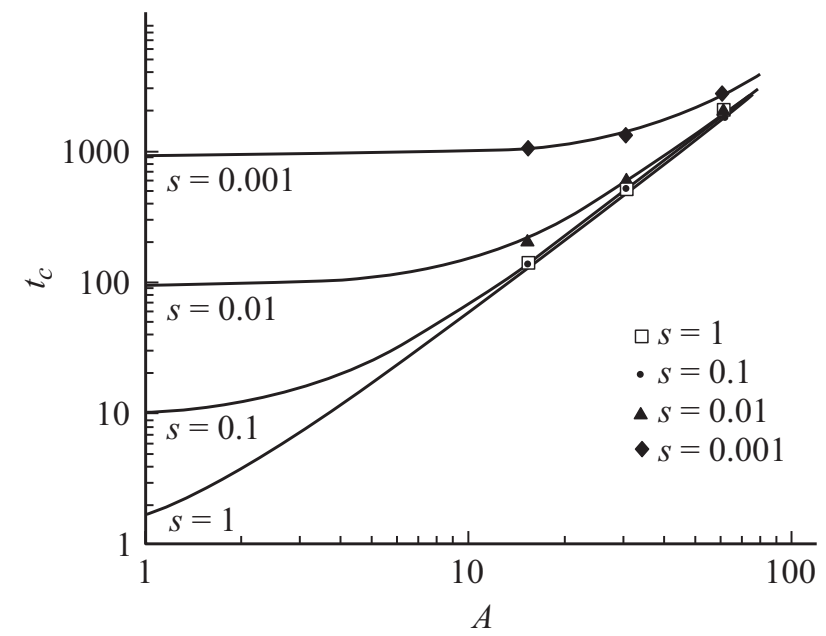

Рис. 7. Аналитические зависимости времени, необходимого для однородного покрытия стенок цилиндрического отверстия, от аспектного отношения при различных значениях коэффициента прилипания, рассчитанные по формуле (29) при $k=3.5$ и $\alpha=1.9$. Значения, отмеченные точками, взяты из работы [5].

зависимости, рассчитанные по формуле (36) (штриховые линии), являются хорошей аппроксимацией кривых, полученных численным решением системы интегродифференциальных уравнений (21).

С другой стороны, следует заметить, что выражение (36) было получено на основе анализа предельных случаев и не учитывает ряд факторов, таких как уменьшение плотности потока частиц за счет вылета из отверстия; увеличение количества отражений по мере заполнения его стенок; поток частиц, отраженных от дна отверстия. Если принять коэффициенты в (36) как свободные параметры, то расхождение между расчетными кривыми и результатами других авторов можно свести к минимуму.

На рис. 7 изображен график аналитической зависимости времени, необходимого для однородного покрытия стенок цилиндрического отверстия, от аспектного отношения при различных значениях коэффициента прилипания, рассчитанные по формуле

$$
t_{c}(A, s)=\frac{n_{0}}{J_{f}}\left(\frac{1}{s} \ln \left(\frac{1}{1-\eta}\right)+k A^{\alpha}\right),
$$

где параметры $k=3.5$ и $\alpha=1.9$ были выбраны для наилучшего соответствия кривых точкам, полученным в работе [5]. Критическое время нормировано на время покрытия плоской поверхности при $s=1$. Как можно видеть из рисунка, при указанных параметрах формула (37) описывает зависимость критического времени от аспектного отношения при различных значениях коэффициента прилипания, определяемого температурой ALD-процесса, практически совпадая с результатами длительного численного эксперимента [5]. 


\section{Заключение}

В работе применен кинетический подход, описывающий рост пленки на стенках цилиндрического отверстия, методом атомно-слоевого осаждения. Модель позволяет предсказать пространственный профиль получаемого покрытия, а также рассчитать минимальное время, необходимое для конформного покрытия стенок $3 D$-структуры (критическое время). На основании анализа расчетных кривых зависимости критического времени покрытия стенок отверстия от аспектного отношения при различных значениях коэффициента прилипания были выявлены два асимптотических режима, соответствующих мелким $\left(1 / s \gg A^{2}\right)$ и глубоким $\left(1 / s \ll A^{2}\right)$ отверстиям. Для мелких отверстий критическое время подачи прекурсора примерно постоянно и соответствует времени заполнения плоской поверхности. В случае глубоких отверстий критическое время примерно пропорционально квадрату аспектного отношения и фактически не зависит от коэффициента прилипания. Результаты качественно и количественно согласуются с исследованиями [5-8], использовавшими иные модели ALD-роста.

На основании выявленных асимптотических закономерностей была получена аналитическая зависимость критического времени, необходимого для однородного покрытия стенок отверстия как функции аспектного отношения и коэффициента прилипания.

\section{Список литературы}

[1] Gardner D.S., Holzwarth C.W., Liu Y. // Electron Devices Meeting (IEDM). 2014. IEEE International. doi 10.1109/IEDM.2014.7047009

[2] Atomic Layer Deposition of Nanostructured Materials/ Ed. by N. Pinna, M. Knez. Wiley-VCH Verlag \& Co. KGaA, 2012. $435 \mathrm{p}$

[3] Gordon R.G., Hausmann D., Kim E., Shepard J. // Chem. Jap. Depos. 2003. Vol. 9. N 2. P. 73-78.

[4] Rose M., Bartha J.W. // Appl. Surf. Sci. 2009. Vol. 256. P. 6620-6623.

[5] Rose M., Bartha J.W., Endler I. // Appl. Surf. Sci. 2010. Vol. 255. P. 3778-3782.

[6] Knoops H.C.M., Langereis E., van de Sanden M.C.M., Kessels M.M. // J. Electrochem. Soc. 2010. Vol. 57. N 12. P. G241-G249.

[7] Yanguas-Gil A., Elam J.W. // ECS Transactions. 2011. Vol. 41. N 2. P. $169-174$.

[8] Yanguas-Gil A., Elam J.W. // Chem. Vap. Depos. 2012. Vol. 18. P. 46-52.

[9] Wang W.B., Abelson J.R. // J. Appl. Phys. 2014. Vol. 116. P. 116-124.

[10] Фадеев А.В., Млконьких А.В., Руденко К.В. // ЖТФ. 2018. Вып. 88. № 2. С. 243-250.

[11] Розанов Л.М. Вакуумная техника. М.: Высшая школа, 1990. $320 \mathrm{c}$. 